\title{
Post-Menopausal Woman with Frontal Hairline Recession
}

\author{
Paulo Müller Ramos ${ }^{\text {a }}$ Maria Teresa Nakandakari ${ }^{\mathrm{b}}$ \\ ${ }^{a}$ Dermatology Department, São Paulo State University, UNESP, Botucatu, Brazil; b Private Practice, Bauru, Brazil
}

\section{Question}

A 69-year-old woman presented with a one-year history of hair loss affecting the hairline and eyebrows. During a previous appointment with a dermatologist, a skin biopsy had been taken, which had resulted in the diagnosis of alopecia areata (AA). The patient had been using topical tacrolimus $0.1 \%$ and had received intralesional injections of corticosteroids but without benefit. She also complained of generalized itching, but with no skin lesions.
On clinical examination, the patient presented alopecia affecting the anterior hairline (frontal and temporal) and loss of eyebrows (more pronounced on the right side). There were no skin lesions, such as papules or scale, in the affected areas (Fig. 1). Dry dermoscopy revealed slightly diffuse erythema with low hair density, mild perifollicular scaling, black dots, exclamation hairs, and loss of some follicular openings (Fig. 2a). On immersion dermoscopy, yellow dots, vellus hairs, and circle hairs were evident (Fig. 2b).

What is your diagnosis?

\section{KARGER}

(c) 2019 S. Karger AG, Basel

E-Mail karger@karger.com

www.karger.com/sad
Paulo Müller Ramos 
Fig. 1. Alopecia affecting the anterior hairline (frontal and temporal) and loss of eyebrows (more pronounced on the right side).

Fig. 2. a Dry dermoscopy presented slightly diffuse erythema with low hair density, mild perifollicular scaling, black dots, exclamation hairs, and loss of some follicular openings. b Immersion dermoscopy presented yellow dots, vellus hairs, and circle hairs.
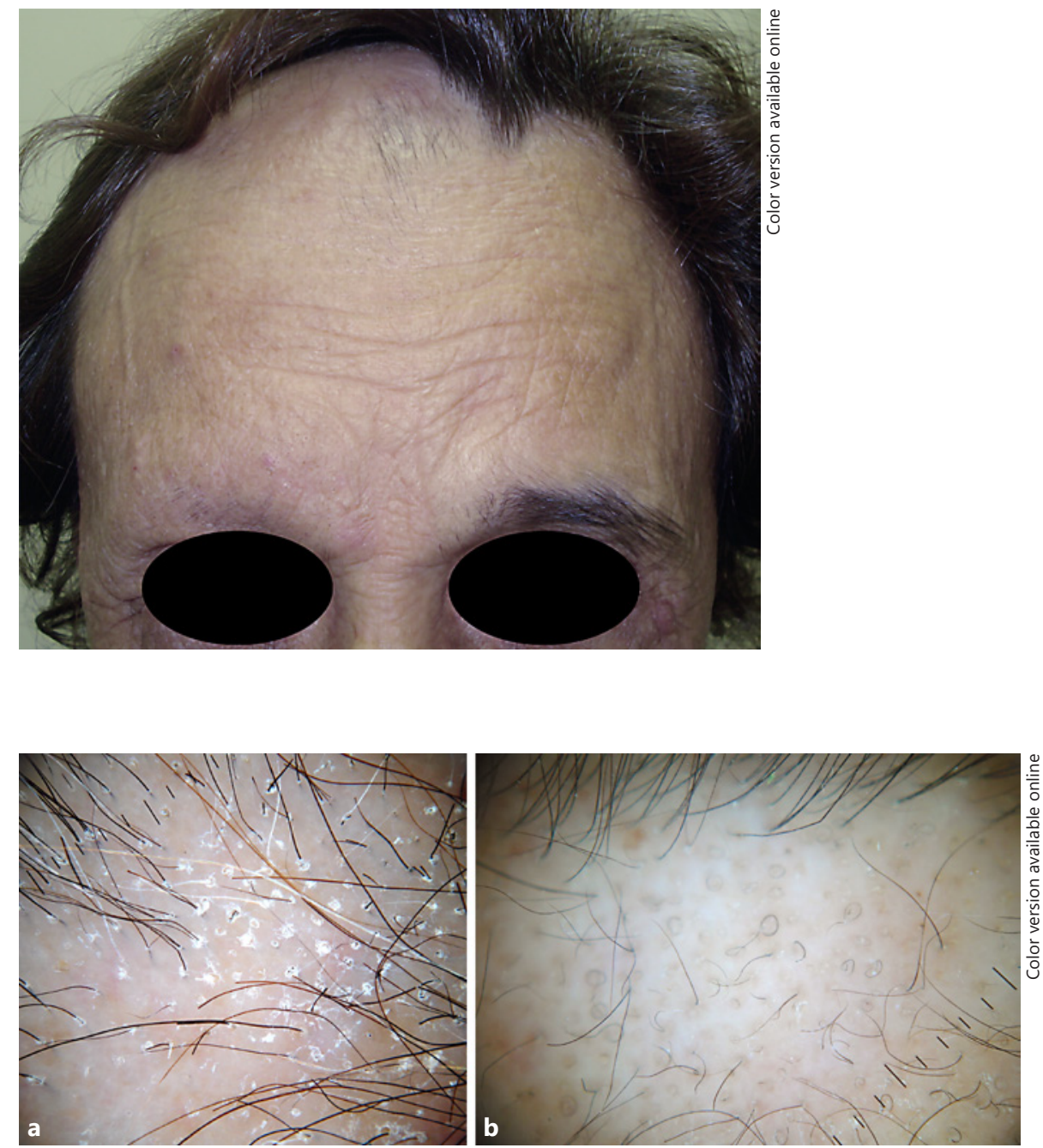


\section{Answer}

\section{Folliculotropic Mycosis Fungoides}

\section{Discussion}

For a post-menopausal woman presenting with hair loss of the anterior hairline and eyebrows with follicular scaling, the most straightforward diagnosis would be frontal fibrosing alopecia; however, the presence of yellow dots, vellus hairs, and circle hairs revealed by dermoscopy do not support this hypothesis. These dermatoscopic signs are consistent with her previous diagnosis of AA; on the other hand, the diffuse erythema, perifollicular scaling, and loss of follicular openings were not expected in AA. Considering the lack of full clinical, dermatoscopic, and histologic correlation, a new biopsy was taken of the frontal region of the scalp at the border of the alopecic area.

In a second histologic evaluation, sebaceous glands were present, and there were no signs of scarring alopecia. There was a mononuclear infiltrate of atypical cells with a large hyperchromatic nucleus damaging the hair follicles and sweat glands, and folliculotropism in the presence of Pautrier's abscess (Fig. 3). Histological sections stained with colloidal iron demonstrated mucin depositions. Immunohistochemistry returned CD4 positive, CD8 negative, and focally positive CD30.

Alopecia is an unusual manifestation in patients with mycosis fungoides (MF). In a retrospective study of 1,150 patients with MF, just $2.5 \%$ presented with alopecia [1]. Of these patients, $66 \%$ presented with alopecia associated with MF lesions, with clear epidermal changes such as patches, plaques, and erythroderma. Thirty-four percent presented with hair loss in an areata-like pattern. Epidermal changes, when present in these areas of baldness, were limited to mild erythema or scaling.

A dermoscopic analysis of areata-like MF has been previously described presenting with follicular or diffuse scaling, a reduced number of follicular openings with broken hairs, and keratotic filiform spicules [2]. In this case, we found dermatoscopic signs that would be expected both in frontal fibrosing alopecia (perifollicular scaling and loss of follicular openings) and AA (yellow dots, exclamation hairs, and circle hairs).

During a previous appointment with another dermatologist, a skin biopsy had been taken from our patient which had resulted in the diagnosis of AA. On the horizontal section of the skin biopsy, areata-like MF can pres-

Post-Menopausal Woman with Frontal Hairline Recession

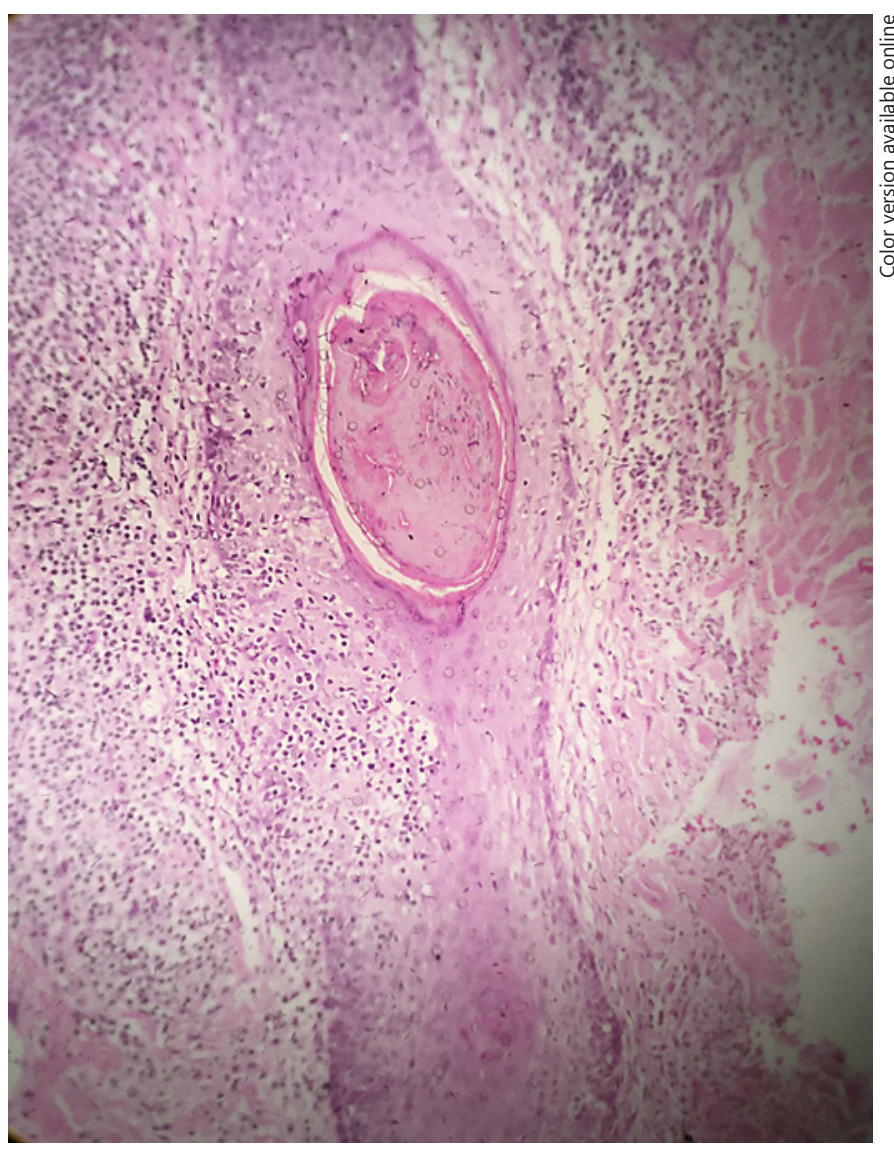

Fig. 3. Histologic evaluation revealed a mononuclear infiltrate of atypical cells with a large hyperchromatic nucleus damaging the hair follicles and sweat glands, and folliculotropism in the presence of Pautrier's abscess.

ent as peribulbar lymphocytic infiltrate, mimicking AA [3]. Probably, this led to the initial misdiagnosis of AA in this case. Multiple horizontal sections from the bulb to the infundibulum could help to avoid this diagnostic error.

Folliculotropic MF has distinct clinical features with high rates of facial involvement and a poor prognosis compared to MF [4]. In this case, the initial therapy consisted of psoralen plus UVA (PUVA) with good response. After a few months, our patient was lost to follow-up.

\section{Statement of Ethics}

Our patient has given her consent to have details and photos of her case published. 


\section{Disclosure Statement}

The authors declare no conflicts of interest.

\section{Keywords}

Mycosis fungoides - Alopecia areata $\cdot$ Areata-like pattern . Ophiasis

\section{References}

1 Bi MY, Curry JL, Christiano AM, Hordinsky MK, Norris DA, Price VH, et al. The spectrum of hair loss in patients with mycosis fungoides and Sézary syndrome. J Am Acad Dermatol. 2011 Jan;64(1):53-63.

2 Miteva M, El Shabrawi-Caelen L, Fink-Puches R, Beham-Schmid C, Romanelli P, Kerdel $\mathrm{F}$, et al. Alopecia universalis associated with cutaneous T cell lymphoma. Dermatology. 2014;229(2):65-9.
3 Iorizzo M, El Shabrawi Caelen L, Vincenzi C, Misciali C, Tosti A. Folliculotropic mycosis fungoides masquerading as alopecia areata. J Am Acad Dermatol. 2010 Aug;63(2):e50-2.

4 Gerami P, Rosen S, Kuzel T, Boone SL, Guitart J. Folliculotropic mycosis fungoides: an aggressive variant of cutaneous T-cell lymphoma. Arch Dermatol. 2008 Jun; 144(6): $738-46$. 